Chirurg 2012 $\cdot 83: 739$

DOI 10.1007/s00104-012-2343-5

Online publiziert: 10. August 2012

(c) Springer-Verlag 2012
O. Strobel · M.W. Büchler

Klinik für Allgemein-, Viszeral- und Transplantationschirurgie, Universität Heidelberg

\section{Laparoskopische Pankreaslinksresektion}

\section{Ist sie dem offenen Operationsverfahren überlegen?}

\section{Originalpublikation}

Venkat R, Edil BH, Schulick RD et al (2012) Laparoscopic distal pancreatectomy is associated with significantly less overall morbidity compared to the open technique: a systematic review and meta-analysis. Ann Surg 255:1048-1059

\section{Hintergrund und Fragestellung}

In der Pankreaschirurgie werden zunehmend minimal-invasive Verfahren eingesetzt. Insbesondere die laparoskopische Pankreaslinksresektion (LPLR) wird in immer mehr Kliniken durchgeführt. Es ist aber unklar, ob und bei welchen Indikationen die LPLR der offenen Pankreaslinksresektion (OPLR) überlegen ist. In einem systematischen Review mit Metaanalyse gingen Venkat et al. dieser Frage nach.

\section{Methoden}

Es wurden 18 Studien mit insgesamt 1814 Patienten identifiziert, die Ergebnisse von LPLR und OPLR verglichen (773 vs. 1041 Patienten). Alle Studien waren retrospektiv und nicht kontrolliert. Bei 50\% der Studien war Malignität ein Ausschlusskriterium. Bei nur 10 Studien (56\%) wurde eine Matchedpair-Analyse bezüglich der pathologischen Diagnose durchgeführt. Bei Konversionsraten von bis zu $25 \%$ blieb unklar, ob in den einzelnen Studien eine Intention-to-treatAnalyse durchgeführt wurde. OutcomeParameter waren verschiedene Kenngrößen des intra- und perioperativen Verlaufs. Onkologische Zielgrößen waren der R-Status und die Anzahl analysierter Lymphknoten.

\section{Ergebnisse}

Die LPLR war im Vergleich zur OPLR mit einem geringeren Blutverlust, einer kürzeren Krankenhausverweildauer sowie einer geringeren Gesamtmorbidität und einer geringen Rate an Wundinfektionen assoziiert. Es ergaben sich keine Unterschiede in der Operationsdauer, in der Rate an Pankreasfisteln und in der Mortalität. Basierend auf 4 Studien war kein Unterschied bezüglich des R-Status nachweisbar. Die Anzahl analysierter Lymphknoten war in 2 Studien nach OPLR höher und in 2 Studien nach LPLR und OPLR vergleichbar.

\section{Schlussfolgerung}

Die Autoren folgern dass die LPLR im Vergleich zur OPLR bei vergleichbarer Operationsdauer zu weniger Blutverlust, kürzerer Krankenhausverweildauer und geringerer Morbidität führt. Aufgrund der vergleichbaren Ergebnisse zum R-Status folgern die Autoren, dass die Durchführung einer LPLR auch bei selektierten $\mathrm{Pa}$ tienten mit Malignom sinnvoll sei.

Die Ergebnisse dieser Metaanalyse sind jedoch mit Vorsicht zu interpretieren:

- Aufgrund der retrospektiven Natur der analysierten Studien und des Ausschlusses von Malignität in einem Großteil dieser Studien ist von einem deutlichen Bias durch Selektion leichterer Fälle in das LPLR-Kollektiv auszugehen. Die beiden Kollektive sind bezüglich der wichtigsten Zielparameter wahrscheinlich nicht vergleichbar.
- Angesichts der Konversionsraten und der fehlenden Angaben bezüglich einer Intention-to-treat-Analyse bestehen weitere Bedenken hinsichtlich der Validität.

- Bezüglich der Sinnhaftigkeit einer LPLR bei onkologischen Fällen ist keine fundierte Konklusion möglich, insbesondere weil Follow-up-Daten fehlen.

Fazit

Die LPLR ist nach Durchschreiten einer Lernkurve ein sicheres Verfahren zur Resektion umschriebener benigner Prozesse im Pankreasschwanz. Bei Malignitätsverdacht sollte eine sehr sorgfältige Patientenselektion erfolgen. Die Durchführung einer LPLR darf nicht zu Kompromissen bei der onkologischen Radikalität führen. Wie 2 weitere aktuelle Metaanalysen der vorliegenden retrospektiven Studien lässt auch die Arbeit von Venkat et al. keine sichere Konklusion bezüglich der Vergleichbarkeit von LPLR und OPLR zu. Diese Frage kann nur durch gut konzipierte randomisiert kontrollierte Studien beantwortet werden.

\section{Korrespondenzadresse}

PD Dr. O. Strobel

Klinik für Allgemein-, Viszeral- und Transplantationschirurgie, Universität Heidelberg Im Neuenheimer Feld 110, 69120 Heidelberg Oliver.Strobel@med.uni-heidelberg.de

Interessenkonflikt. Der korrespondierende Autor gibt für sich und seinen Koautor an, dass kein Interessenkonflikt besteht. 